\title{
Natural barrier to HIV-1 entry
}

DOI:

10.1038/nrd2342
Although it is known that molecules with anti-HIV activity circulate in human blood, their identification has been hampered by difficulties in the purification and characterization of such compounds. Kirchhoff and colleagues undertook the task of systematically screening a haemofiltrate-derived peptide library of over one million small peptides for natural inhibitors of HIV-1 replication and report the isolation of a novel virus inhibitory peptide (VIRIP) that is active against a wide variety of HIV-1 strains.

VIRIP is a 20 -residue peptide, corresponding to a fragment of the highly abundant serine protease inhibitor $\alpha 1$-antitrypsin, which strongly inhibits HIV-1 infection and transmission in human peripheral blood mononuclear cells (PBMCs). Chemically synthesized VIRIP inhibited PBMC infection by a broad range of HIV strains, including variants that are resistant to current antiretroviral drugs, with similar potency to isolated VIRIP and without causing cytotoxicity.

Functional and structural data show that VIRIP binds to a hydrophobic region of the HIV-1 envelope glycoprotein gp41, preventing membrane fusion and virus entry. Moreover, by substituting a few of VIRIP's amino acids to stabilize the

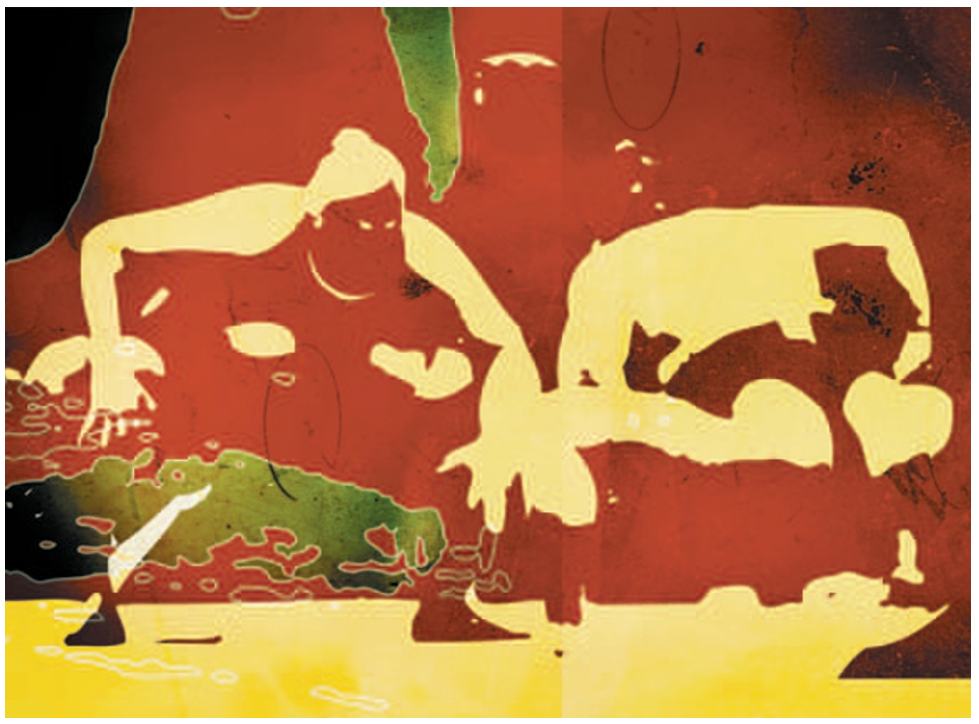

active conformation and to increase the hydrophobic contact area, the authors were able to increase the inhibitory potency of VIRIP by two orders of magnitude.

In contrast to enfuvirtide (also known as T20), the first HIV-1 entry inhibitor to be approved by the FDA, it proved difficult to generate strains of HIV-1 that were resistant to VIRIP derivatives in vitro. This finding indicates that VIRIP binds to a highly conserved region of gp41 and supports the use of VIRIP derivatives against HIV-1 variants that are resistant to other entry inhibitor drugs.
What triggers the generation of VIRIP and its function in the human body remain elusive, but its activity, stability and lack of toxicity all herald optimized VIRIP derivatives as promising candidates for clinical assessment. This study also highlights a region in gp41 as an attractive drug target that may pave the way for the development of a new class of HIV-1 entry inhibitors.

Monica Hoyos Flight

ORIGINAL RESEARCH PAPER Münch, J. et al.

Discovery and optimization of a natural HIV-1 entry inhibitor targeting the gp41 fusion peptide. Cell 129, 263-275 (2007) 